\title{
CORONARY ARTERIOSCLEROSIS AND CALCIFICATION IN INFANCY
}

\author{
BY \\ R. A. SLADDEN \\ From the Department of Morbid Anatomy, Radcliffe Infirmary, Oxford
}

(RECEIVED FOR PUBLICATION SEPTEMBER 27, 1951)

Several reports have appeared in recent years in the American literature dealing with the occurrence of arteriosclerosis and arterial calcification in infancy. This process may affect the coronary arteries in particular, possibly giving rise to myocardial infarction.

Although the condition is mentioned briefly in some textbooks of pathology and paediatrics, it has attracted few reports from this country. The latest of these appears to be that of Lightwood (1932), if that case can be said to have occurred during infancy, the child having died at 27 months. It is probable that some isolated cases have not been reported.

Field (1946) and Stryker (1946) both indexed the available cases up to that time, including Lightwood's case, added personally studied cases, and gave comprehensive reviews on the question of aetiology. Out of a total of 25 cases of arterial calcification referred to by these authors, 22 showed involvement of the coronary arteries. A similar type of case was reported by Andersen in 1945. A year later Lingmark and Murray added a further case, and referred to another case in the literature, that of Scheidegger (1940). Cases have since been reported by Hause and Antell (1947, one case); Prior and Bergstrom (1948, two cases); Menten and Fetterman (1948, three cases). Brief reference was made by Weller (1940) to an apparently similar type of case. Those few reports in the literature which describe arterial calcification in infancy, without the mention of coronary arterial involvement, are not considered here.

It appears to be generally agreed that no single aetiological factor has been identified as the cause of this condition. There is evidence in some cases that renal disease may play an important role ; arterial calcification is known to have occurred in some cases of renal rickets in older children (Shelling and Remsen, 1935 ; Smyth and Goldman, 1934). Even if such cases as those with gross renal pathology (Bryant and White, 1901 ; Andersen and Schlesinger, 1942; Lightwood, 1932; Stryker, Case 4, 1946) are excluded from consideration, there still remains a majority for which no satisfactory aetiology has been found. To this latter group belong the two cases reported below.

Other reputed aetiological factors include syphilis, other infections, allergy, and hypervitaminosis D. 


\section{Clinical Features}

The disease has been reported in a stillborn infant (Stryker, 1946) and in a child of 27 months (Lightwood, 1932).

There are 19 males, 12 females, and two cases of unstated sex in a total of 33 cases, including the two cases reported here.

The family history may be of importance, since Menten and Fetterman (1948) reported the disease in two siblings.

There have been no constant findings of apparent significance in the histories of pregnancy, delivery, or upbringing. Although the administration of vitamin D or calcium supplements to mother or child is referred to in some reports, in only one case do the authors feel justified in incriminating hypervitaminosis D as an aetiological factor (Ross and Williams, 1939).

Once the child becomes ill there is usually a fairly steady downhill course terminating in death in a few days or weeks. Sudden death has been reported. The child refuses its feeds, loses weight, and may begin to vomit. Clinical signs are usually referred to the cardiovascular or respiratory systems, but may be completely absent. Cardiac enlargement, periodic attacks of cyanosis and dyspnoea, enlargement of the liver and spleen, ankle oedema, and ascites may be found. Congestion of the lungs associated with low-grade pyrexia sometimes suggests a diagnosis of bronchopneumonia, which, in fact, often supervenes terminally. Diarrhoea and other terminal infections have also been reported. Bryant and White (1901) reported gangrene of the right foot as a result of arterial occlusion. In the second case reported below it is almost certain that the child was suffering from paroxysmal attacks of angina pectoris. In Case 1 of Prior and Bergstrom (1948) involvement of the meningeal and cerebral vessels gave rise to neurological signs. Radiographs of the limbs in this case, taken during life, were re-examined after death and showed calcification of the limb vessels, but radiological and laboratory investigations are usually of little value. The highest value obtained for serum calcium was in Case 1 of Menten and Fetterman (1948), the level at necropsy being 16.5 mg. $/ 100 \mathrm{ml}$. The serum phosphates were $4.86 \mathrm{mg} . / 100 \mathrm{ml}$.

\section{Pathological Features}

The essential pathological lesion is found in the medium-sized arteries of the body, including the coronary arteries, and consists of fibroblastic proliferation of the intima with calcification occurring in close relationship to the internal elastic lamina and spreading into the medial coat and proliferated intima. There are varying degrees of disorganization of the vessels, obstruction of the lumina and recanalization, round cell, and occasional giant cell or eosinophilic infiltration of the vessel walls. These changes have been reported from nearly all parts of the body, including the brain, lungs, abdominal viscera, and limbs. The aorta may also show some calcification in relation to the elastic fibres of the media. If coronary occlusion occurs, myocardial necrosis and calcification of the affected muscle may take place. The left ventricle and papillary muscles are frequently involved.

Various types of pneumonia have been described in this series.

Parathyroid hyperplasia was reported by Andersen and Schlesinger (1942), probably secondary to bilateral hydronephrosis and congenital hypoplastic kidneys in one case, and polycystic kidneys in the other. Stryker (1946) reported polycystic 
kidneys and multiple congenital abnormalities in his case 4; Bryant and White (1901) recorded congenital urethral dilatation with hydronephrosis, and in Oppenheimer's (1938) Case 2 there was a congenital malformation of the genitalia.

Early or chronic glomerulo-nephritis has been reported in a few cases, and Lightwood (1932) described glomerular and interstitial fibrosis with extensive calcinosis of the kidney in his case of dwarfism.

Andersen (1945) observed extensive aortic involvement associated with cirrhosis and interstitial calcification of the liver. The infant died shortly after birth : no evidence of syphilis was obtained.

\section{Case Reports}

Case 1 (Reg. No. R.I. 5254/39). A boy, aged 18 months, was born normally, three weeks prematurely. He was breast-fed for one year, then weaned on half a cup of cow's milk a day.

Neither mother nor child received vitamin or calcium supplements.

At the age of 14 months he fell and hurt his head, afterwards developing a sore throat and anorexia. Nine weeks later he was admitted to hospital.

On admission he was seen to be a pale, lethargic infant with slight cyanosis of the lips. The heart was found to be enlarged on clinical examination. The liver was palpable one fingerbreadth below the costal margin. One week later the feet became oedematous and remained thus for three days. Numerous intermittent attacks of fever and dyspnoea with some liver enlargement occurred before death, which took place in a final attack. There had been some vomiting and progressive emaciation. The clinical diagnosis was pneumonia.

Post-mortem Examination (P.M. 425/39).-The body was that of an emaciated male infant.

Lungs. - There were signs of chronic congestion, and the lungs were rather indurated.

Heart. - The heart weighed $137 \mathrm{~g}$., and there was a sterile, gelatinous pericardial exudate. Generalized cardiac hypertrophy, especially of the left ventricle, was seen and endocardial thickening, especially in the region of the mitral valve.

Liver.-The liver was enlarged, firm and congested.

Kidneys.-The kidneys were congested, with several recent haemorrhagic cortical infarcts and adherent capsules.

Suprarenal.-Marked congestion in the deep cortical layers was seen.

Histological Examination.-The following organs were examined histologically.

Lungs. - There was a considerable degree of vascular congestion together with early bronchopneumonic changes. Many alveolar macrophages contained vacuoles suggestive of inhalation lipoid pneumonia.

Heart.-Some hypertrophy of myocardial fibres was seen. A branch of the coronary artery showed calcification in relation to the internal elastic lamina and slight irregular intimal proliferation.

Liver.- In the liver there was considerable fatty change, centrilobular congestion and necrosis. Occasional round-cell infiltration of the portal tracts was seen.

Kidneys.-These showed areas of calcification involving the tubules and main renal arterial vessels. Small focal areas of calcification of tubular epithelium were seen, with occasional extrusion of the calcified masses into the lumina of the tubules.

The affected tubules were usually in close relation to glomeruli and were probably of the proximal convoluted type. Very occasional foci of calcification were seen within 
glomeruli of a rather immature type ; the more mature glomeruli were apparently not affected.

Many of the main branches of the renal artery showed irregular plaques of intimal proliferation and deposition of calcium along the underlying internal elastic laminae. These deposits quite often extended into the media forming roughly spherical masses, but there did not appear to be any medial hypertrophy or inflammatory reaction either of the media or intima.

Linear deposits of calcium were occasionally found along the internal elastic laminae of some of the smaller branches of the renal arteries, but arterioles were not affected and the vascular changes were confined to the medulla and pelvic tissues, whereas focal tubular calcification was scattered throughout the renal parenchyma.

Pancreas.-Large arteries in the region of the pancreas showed changes similar to those seen in the renal arteries.

Suprarenals.-Arterial calcification of the type already described was found in the suprarenal arteries, but more striking changes were found in the sinusoidal vessels of the zona reticularis and medulla. The majority of these contained irregular calcified bodies of varying sizes, often laminated, and sometimes containing at one edge a cell nucleus suggestive of an endothelial origin. It appeared that the calcium might have been deposited directly upon, or within, the endothelial lining cells.

The cells of the suprarenal cortex and medulla showed no abnormalities.

The pathological diagnosis was calcification of the coronary arteries and mediumsized arteries of the tongue, pancreas, and kidneys ; calcification of the sinusoids of the suprarenal medulla; myocardial hypertrophy; low-grade bronchopneumonia; acute pericarditis.

Case 2* (Reg. No. R.I. 129655).-A boy, aged 3 months, was born normally after a full term pregnancy. He was breast-fed for three weeks, then given national dried milk and the usual cod-liver oil supplements. Weight was gained steadily. At the age of three months he refused his feed one evening and the same night began crying. Twenty hours later respirations became rapid and gasping. He had two further attacks of dyspnoea, screaming, and evident pain which appeared to be abdominal in origin. The attacks were followed by collapse and cyanosis, the extremities becoming cold and clammy.

On admission to hospital the liver and spleen were both palpable. No pus cells or red cells were found in the urine. Radiographs of the chest and abdomen and a barium enema showed no definite lesion.

In view of the severe pain, presumably of abdominal origin, laparotomy was performed but no abnormality was found. Four hours after operation and two days after the beginning of the illness the child suddenly collapsed and died.

The parents were both alive and well. There was one healthy sibling aged 3 years. The mother had not received vitamin D therapy.

Post-mortem Examination (P.M. 391/50). - The body was that of a normally developed male child.

Lungs.-Patchy areas of atelectasis were seen.

Liver and Spleen.-The liver and spleen were congested.

Arteries.-The mesenteric, femoral, and tibial arteries were calcified; numerous arteries in the region of the thyroid gland were enlarged, calcified, and tortuous.

* The radiological aspects of this case have been reported briefly by Boldero (1951). 
Heart.-The heart, together with the aortic arch and the roots of the great vessels, weighed $60 \mathrm{~g}$. The heart measured $7 \times 4.5 \times 4.3 \mathrm{~cm}$. and the left ventricular wall measured from 0.8 to $1.3 \mathrm{~cm}$. in thickness. The coronary arteries were conspicuous, hard and tortuous (Fig. 1), and there was a small subepicardial haemorrhage around the circumflex branch of the left coronary artery. There was necrosis of the anterior papillary muscle of the mitral valve and of a small area of the interventricular septum. Radiography of the viscera revealed calcification of the hepatic, pulmonary, splenic, pancreatic, renal, and suprarenal arteries.

The majority of the calcified vessels showed irregular thickening with a somewhat beaded appearance.

The parathyroid glands could not be identified on naked-eye examination. Other organs appeared natural apart from the changes mentioned above. Blood was taken for serum calcium estimation and Wassermann and Kahn reactions.

Histological Examination.-Blocks of tissue were taken from the principal viscera, and also from the carotid, femoral, tibial, brachial, and mesenteric arteries. Fixation was carried out in Gros and Lehan's buffer fixative and in chilled acetone.

All blocks were stained by haematoxylin and eosin ; many by orcein and toluidine blue for elastica and the periodic-acid-Schiff method for polysaccharides.

Gomöri's alkaline phosphatase stain and Von Kossa's stain for calcium were applied to several blocks of the arterial tissue.

Lungs.-Early bronchopneumonic changes were seen. One of the main branches of the pulmonary artery showed scattered linear zones of calcification along the elastic fibres of the media. In general the intima was normal in appearance, but in one area there was some subendothelial fibroblastic proliferation and underlying calcification of the internal elastic lamina.

Heart.- The intima of the coronary arteries (Fig. 2) was generally thickened by subendothelial fibroblastic proliferation, while a few small elastic fibres were seen in its deeper layers. Calcification was seen along the internal eiastic lamina which in places was interrupted by a larger plaque of calcification. Spicules of calcium extended radialiy into the intima. The media, which was relatively thin, showed some diffuse deposition of calcium. As a result of the intimal thickening the lumen of the affected artery was considerably reduced. The myocardium of the left ventricle showed some hypertrophy, and in the interventricular septum and papillary muscles areas of ischaemic necrosis and calcification, surrounded by diffuse fibrosis and some inflammatory reaction (Fig. 4), were seen. The inflammatory cells were chiefly polymorphonuclear leucocytes, including a moderate number of eosinophils.

Liver.-A slight degree of centrilobular congestion was present. The hepatic cells showed no significant changes. There was some periportal acute inflammatory cell infiltration. The larger branches of the hepatic artery were the only ones apparently affected by the process of calcification.

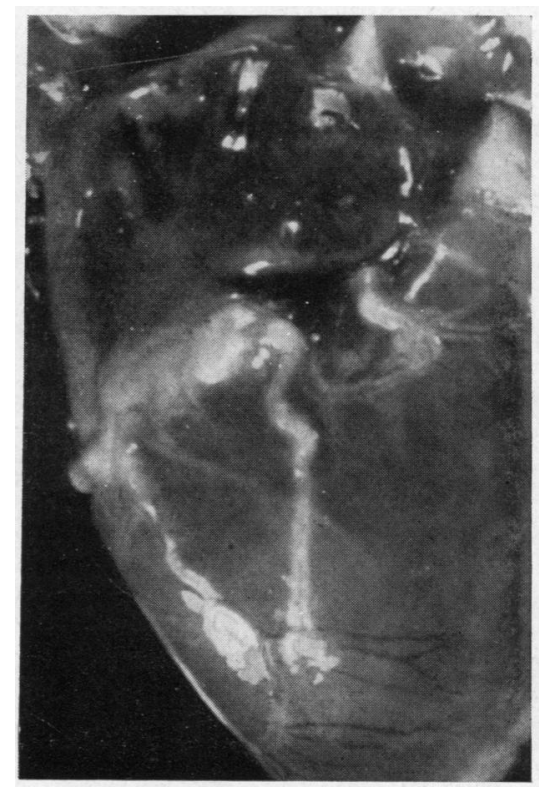

FIG. 1.-Case 2. Heart, showing prominent and tortuous branches of the right coronary artery. 
Spleen.-The spleen was congested, with prominent follicles and a moderately cellular pulp. A fairly small branch of the splenic artery showed focal calcification of the media without intimal thickening.

Kidneys.-With the exception of a very occasional focus of calcification of tubular epithelium, the renal parenchyma exhibited no significant pathological changes. Many of the larger branches of the renal arteries, however, showed focal areas of calcification of the media and internal elastic lamina with some overlying intimal proliferation and occasional calcification of the proliferated intima itself.

Suprarenals.-The cortex and medulla showed no pathological changes and no calcification of the medullary sinusoids was seen.

One of the suprarenal arteries (Fig. 3) showed almost complete obliteration of the lumen by intimal proliferation and calcification. Two multinucleate giant cells were seen at the periphery of the calcified zone. The media appeared to have escaped calcification, but both the media and the intima showed a moderately intense infiltration by eosinophil leucocytes.

Aorta.-An occasional small area of calcification was found in the medial coat. The intima was somewhat irregular in thickness and at one or two points there was fragmentation of the internal elastic lamina with very early deposition of calcium.

Other Blood Vessels.-Changes similar to those already described were found in the femoral, tibial, and mesenteric arteries, and in the arteries of the thyroid region. Some round cell infiltration surrounded the plaques in the femoral artery and a few foreignbody giant cells were also seen.

Calcification was very marked in the thyroid arteries, but was nowhere as advanced as that seen in the suprarenal artery.

Other Organs.-The remaining viscera, the brain and cerebral vessels, and the femoral and sternal bone marrow showed no significant pathological changes. The parathyroid glands were not identified microscopically.

The presence of calcium was confirmed by the use of Von Kossa's stain. It was not possible to demonstrate the presénce of phosphatases in the arterial walls by the use of Gomöri's stain.

The serum calcium was $8.9 \mathrm{mg} . / 100 \mathrm{ml}$. The Wassermann and Kahn reactions were negative.

The pathological diagnosis was calcification of the pulmonary, coronary, hepatic, splenic, mesenteric, pancreatic, renal, suprarenal, thyroid, femoral, and tibial arteries, and of the aorta; myocardial infarction with calcification; occasional calcification of renal tubular epithelium ; early bronchopneumonia.

\section{Discussion}

The intimal changes seen are presumably secondary to the calcification so that from a morphological standpoint this process of calcification is typically metastatic, there being no evidence of pre-existing arterial disease. The rapidity of calcification of the ischaemic myocardium, however, suggests an unusual facility for dystrophic calcification. If the current theories of aetiology, namely, either a disturbance of the ground-substance of the arterial walls or a defect in the elastica, have any basis, then the calcification may be regarded as dystrophic and failure to demonstrate known causes of metastatic calcification is not surprising. These causes in man have been listed by Mulligan (1947) as bone disease, primary parathyroid neoplasm, chronic renal disease, and hypervitaminosis $D$.

The occurrence of the disease in siblings, reported by Menten and Fetterman (1948), together with some other cases suggestive of a familial incidence, might 


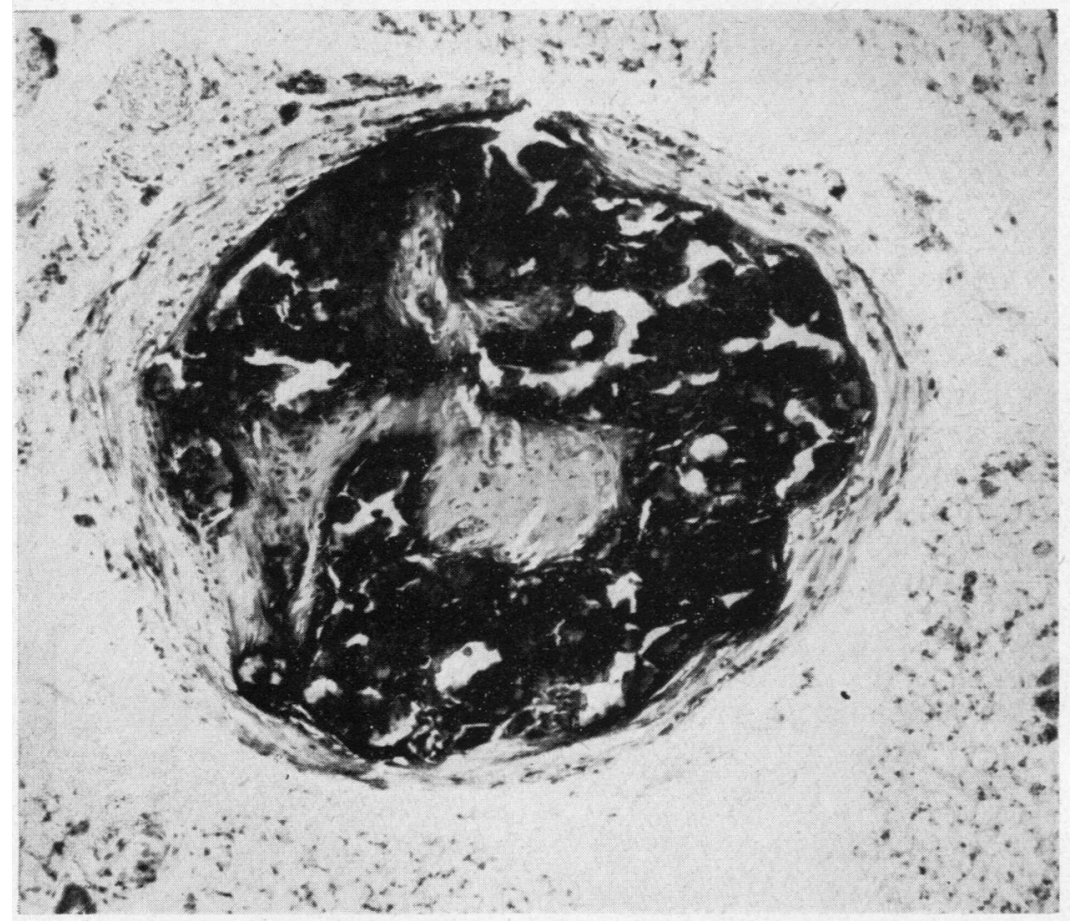

Fig. 3

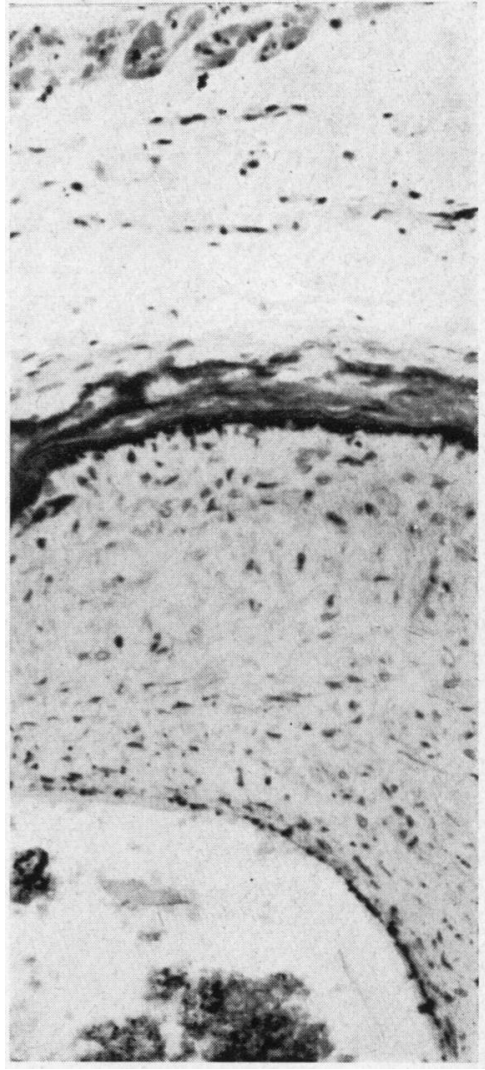

FIG. 2

Fig. 2.-Case 2. Section through left coronary artery near its origin, showing fibroblastic proliferation of the intima and calcification around the internal elastic lamina. (H. and E.) $\times 170$.

FIG. 3.-Case 2. Section of suprarenal artery and surrounding fatty tissue, showing almost complete obliteration of the arterial lumen by intimal proliferation and calcification. (H. and E.) $\times 85$.

FIG. 4.-Case 2. Section of papillary muscle of heart, showing myocardial necrosis and calcification. (H. and E.) $\times 20$.

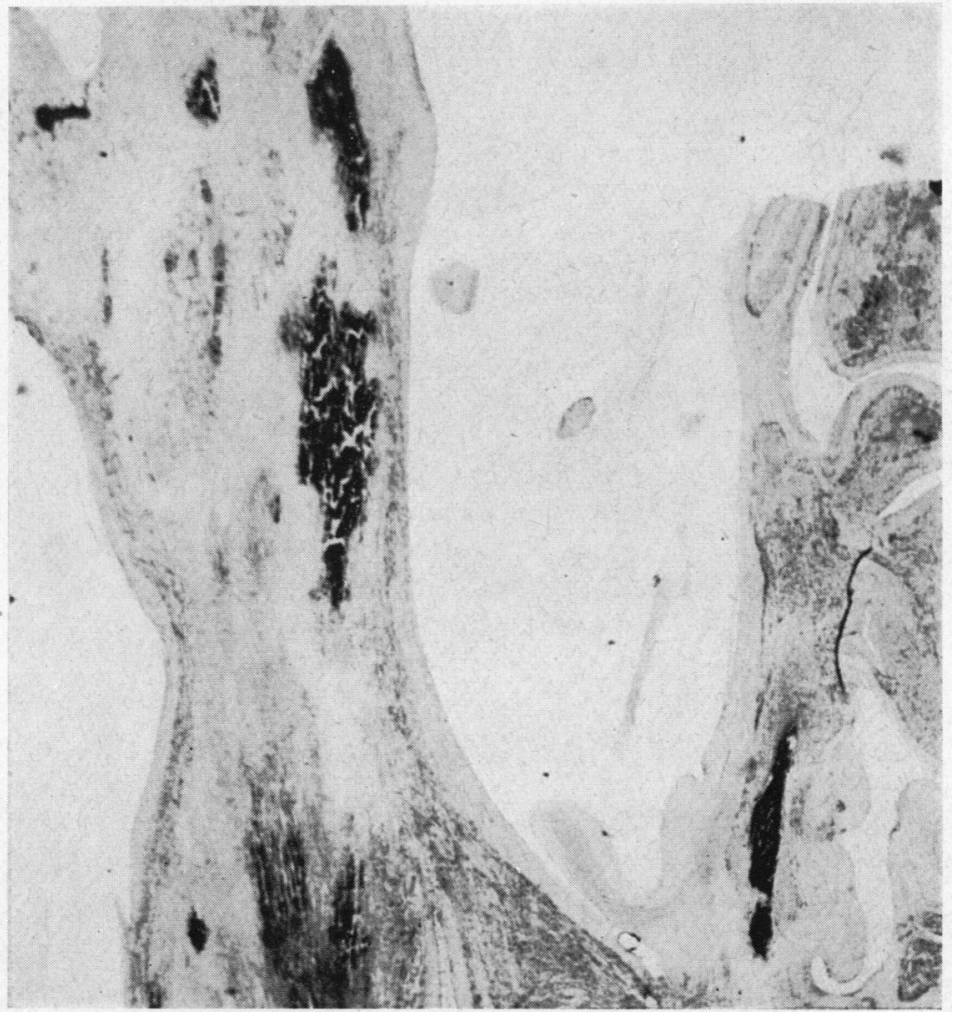

FIG. 4 
favour the theory of some inherent biochemical aberration in ground-substance or elastica. Certainly the process of calcification appears to be closely related to the elastica of the arterial walls.

Minkowski (1947) has studied the formation of intimal cushions and the elastic hyperplasia which not infrequently occurs in the coronary arteries of infants, regarding infection as an important factor. He did not observe calcification in the coronary arteries, but the lability of the arteries in early infancy may play some part in the pathogenesis of the condition under discussion.

As noted by Stryker (1946), the similarity of this condition to the calcification of arteries seen occasionally in young subjects is also of interest. The latter process is seen sometimes in arteries of the thyroid region in otherwise healthy individuals. In Case 2 described above calcification in this area was very marked.

Increased recognition of this disorder may perhaps enable the clinical or radiological diagnosis to be made, and thereby lead to fuller biochemical investigations during life and to prompt anatomical and histochemical studies.

I wish to thank Dr. A. H. T. Robb-Smith for the valuable advice and assistance he has given, and for permission to use the post-mortem report on the first case ; Dr. V. Smallpeice for permission to publish both cases ; and Mr. R. G. Hill for the photography.

\section{REFERENCES}

Andersen, D. H., and Schlesinger, E. R. (1942). Amer. J. Dis. Child., 63, 102.

Andersen, S. R. (1945). Acta path. microbiol. scand., 22, 180.

Boldero. J. L. (1951). Brit. J. Radiol., 24, 43.

Bryant, J. H., and White, W. H. (1901). Guy's Hosp. Rep., 55, 17.

Field, M. H. (1946). Arch. Path., Chicago, 42, 607.

Hause, W. A., and Antell, G. J. (1947). Ibid., 44, 82. (Corr. p. 438).

Lightwood, R. (1932). Arch. Dis. Childh., 7, 193.

Lingmark, I., and Murray, U. (1946). Nord. Med., 29, 183.

Menten, M. L., and Fetterman, G. H. (1948). Amer. J. clin. Path., 18, 805.

Minkowski, W. L. (1947). Amer. J. med. Sci., 214, 623.

Mulligan, R. M. (1947). Arch. Path., Chicago, 43, 177.

Oppenheimer, E. H. (1938). Johns Hopk. Hosp. Bull., 63, 261.

Prior, J. T., and Bergstrom, V. W. (1948). Amer. J. Dis. Child., 76, 91.

Ross, S. G., and Williams, W. E. (1939). Ibid., 58, 1142.

Scheidegger, S. (1940). Frankf. Z. Path., 54, 442. Cited by Lingmark and Murray.

Shelling, D. H., and Remsen, D. (1935). Johns Hopk. Hosp. Bull., 57, 158.

Smyth, F. S., and Goldman, L. (1934). Amer. J. Dis. Child., 48, 596.

Stryker, W. A. (1946). Amer. J. Path., 22, 1007.

Weller, C. V. (1940). Ibid., 16, 666. 